\title{
Intra-alveolar macrophage numbers in current smokers and non-smokers: a morphometric study of tissue sections
}

\author{
W A H Wallace, M Gillooly, D Lamb
}

\begin{abstract}
Background The alveolar macrophage is believed to be important in the defence of the lung and possibly in the pathogenesis of lung disease. Cell counts in bronchoalveolar lavage fluid have suggested that smokers have an increased number of alveolar macrophages but have not enabled the number to be related to a measure of lung structure.
\end{abstract}

Methods The number of alveolar macrophages was counted in histological sections from lung resection specimens from a group of smokers and nonsmokers. The results were related to a measurement of lung structure obtained by means of an automated morphometric technique and expressed in terms of units of lung volume or of lung surface area.

Results The smokers had a significantly increased number of alveolar macrophages per unit lung volume and per unit surface area, though the relative increase was less than has appeared from bronchoalveolar lavage studies. When smokers and non-smokers with similar lung structure were compared the smokers had more alveolar macrophages, indicating that smoking and not loss of lung structure is responsible for the increase.

Conclusions Smokers had more alveolar macrophages than non-smokers when the number was expressed quantitatively with respect to the underlying architecture. Changes in cell populations postulated to be important in the pathogenesis of disease within the lung should be related to lung architecture because this may vary considerably between individuals.

The alveolar macrophage is important in the defence of the lung from external factors such as microbes, dust, and other foreign material. ${ }^{1}$ Its phagocytic capabilities provide the first line of defence against these agents in the distal lung and its importance is illustrated by the clinical sequelae observed in patients with abnormal phagocyte function. ${ }^{2}$ In more recent years it has been shown that as well as having a defensive role in the lung the alveolar macrophage may be important in the pathogenesis of pulmonary disease. ${ }^{3}$ Release of enzymes and chemical mediators from alveolar macrophages may result in pulmonary fibrosis, ${ }^{4}$ and some believe that the alveolar macrophage may have an important role in the pathogenesis of emphysema. ${ }^{5}$

If the alveolar macrophage plays a part either actively or protectively in pulmonary disease it is relevant to assess their number in a quantitative manner. Studies of alveolar macrophage numbers in bronchoalveolar lavage fluid have shown a greater number in smokers than in non-smokers, ${ }^{6-12}$ but have been unable to quantify the increase in terms of lung architecture.

For a quantitative assessment alveolar macrophages must be related in a linear manner to lung volume or lung surface area or both. It has recently been shown that lung architecture can be expressed in terms of airspace surface area per unit lung volume with automated techniques. ${ }^{13-15}$

In this paper we have studied the alveolar macrophage number in relation to unit lung volume and unit surface area in a group of smokers and non-smokers.

\section{Methods \\ PATIENTS}

Surgical resection specimens were obtained from patients undergoing pulmonary surgery for small peripheral lesions at the City Hospital, Edinburgh. Smoking histories were obtained by questionnaire: twelve patientsseven of them men-said that they were current smokers (age 46-66, mean $59 \cdot 2$ years) and nine-six of them men-that they were non-smokers (age 21-69, mean 52.6 years); these included two who had given up smoking more than six months before. All patients had to be fit for a thoracotomy, so patients with severe emphysema were excluded.

The specimens were carefully selected, all having peripheral lesions and no evidence of proximal bronchial obstruction. The specimens from smokers consisted of four pneumonectomy and eight lobectomy specimens. Eleven patients had small peripheral bronchial carcinomas and one had a small, localised area of resolving aspiration pneumonia. The specimens from non-smokers consisted of one pneumonectomy and eight lobectomy specimens (two bronchial carcinomas, two pulmonary carcinoid tumours, two metastatic tumours, one chondromatous hamartoma, one associated lobar sequestration, 
and one localised area of scarring thought on clinical grounds to be a peripheral tumour).

The specimens were transferred within two hours to the laboratory, where they were inflated and fixed transbronchially at $25 \mathrm{~cm}$ pressure with $10 \%$ formol saline. After 24 hours the lungs were serially sliced at $1 \mathrm{~cm}$ intervals in the parasagittal plane. After removal of diagnostic material six random $2 \times 2 \mathrm{~cm}$ blocks were taken, the segment containing the lesion being avoided. None of the blocks showed any evidence of the lesion or of any pathological process resulting from it. The tissue was processed and embedded in glycol methacrylate resin. The blocks were cut into 3 $\mu \mathrm{m}$ sections and stained with haematoxylin and eosin. One further block was taken for processing in paraffin wax.

\section{MEASUREMENT OF LUNG ARCHITECTURE}

Airspace wall surface area per unit volume (AWUV) was measured on the histological sections of lung tissue with an automated scanner, the fast interval processor. ${ }^{15}$ This is an automated device for measuring the mean distance between alveolar walls, expressed as the mean linear intercept $(\mathrm{Lm})$. This figure (Lm) can be used to calculate surface area by using the formula ${ }^{16}$

$$
\text { Surface area }\left(\mathrm{mm}^{2}\right)=\frac{2 V}{L m}
$$

AWUV is the surface area of alveolar walls per unit volume of lung tissue (that is, $V=1 \mathrm{~mm}^{3}$ ) and the above formula therefore becomes

$$
\operatorname{AWUV}\left(\mathrm{mm}^{2} / \mathrm{mm}^{3}\right)=\frac{2}{\mathrm{Lm}}
$$

In this study AWUV was measured on all six haematoxylin and eosin sections from each case and the mean of these values was used. Correction factors for shrinkage artefact were not required as glycol methacrylate resin was used as the embedding medium. ${ }^{17}$

\section{ALVEOLAR MACROPHAGE NUMBERS}

Identification of alveolar macrophages was by morphological examination, at $250 \times$ magnification, of the same $3 \mu \mathrm{m}$ glycol methacrylate sections stained with haematoxylin and eosin. Previously paraffin embedded sections from the same cases had been stained with antiserum to epithelial membrane antigen to exclude significant pneumatocyte desquamation. Alveolar macrophages were counted on randomly selected $1 \mathrm{~mm}^{2}$ fields identified by number coordinates with an England Finder (Graticules Ltd, Tonbridge, Kent). For each case a mean macrophage score per $1 \mathrm{~mm}^{2}$ field was calculated. Using 30 fields per case was shown to give a running mean that did not vary by more than $5 \%$.

One hundred random macrophages from each case were then measured with a digitising tablet and the mean cell volume was obtained by the method of Lindberg and Vorwerk. ${ }^{18}$ From this the mean alveolar macrophage diameter for each case was calculated on the assumption that the cell was approximately spherical. The alveolar macrophage number per $\mathrm{mm}^{3}$ lung tissue was thus

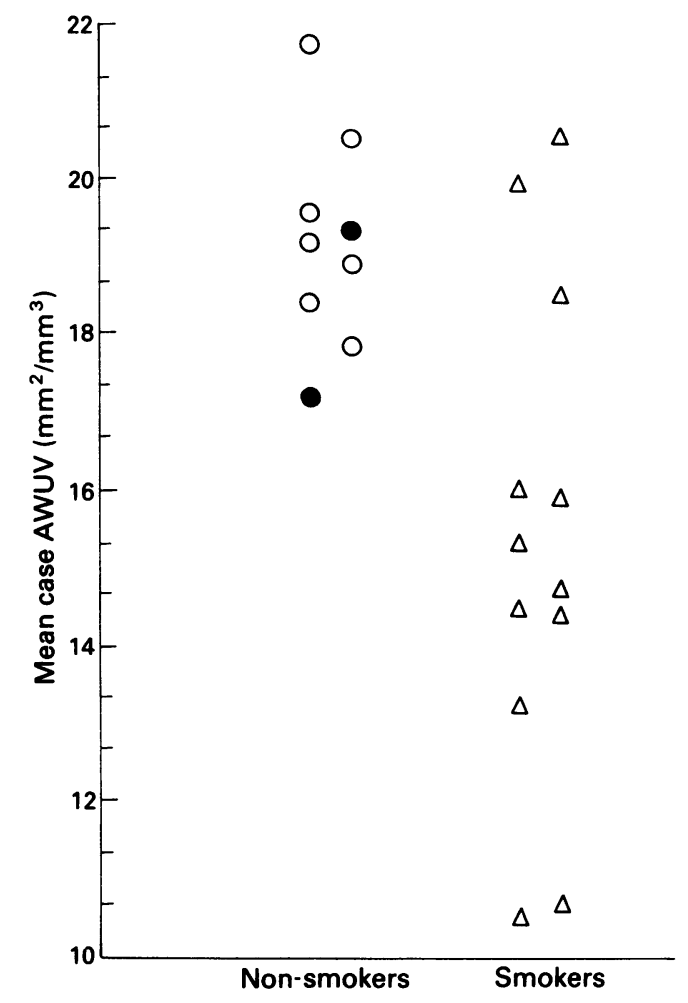

Figure 1 Lung architecture as measured by airspace Figure 1 Lung architecture as measured by airspace
wall surface area per unit volume $\left(A W U V ; \mathrm{mm}^{2} / \mathrm{mm}^{3}\right)$ in the two groups studied. The two ex-smokers are indicated by 0 . The smoking group shows a significant $(p<0.05)$ reduction in $A$ WUV by comparison with the non-smokers.

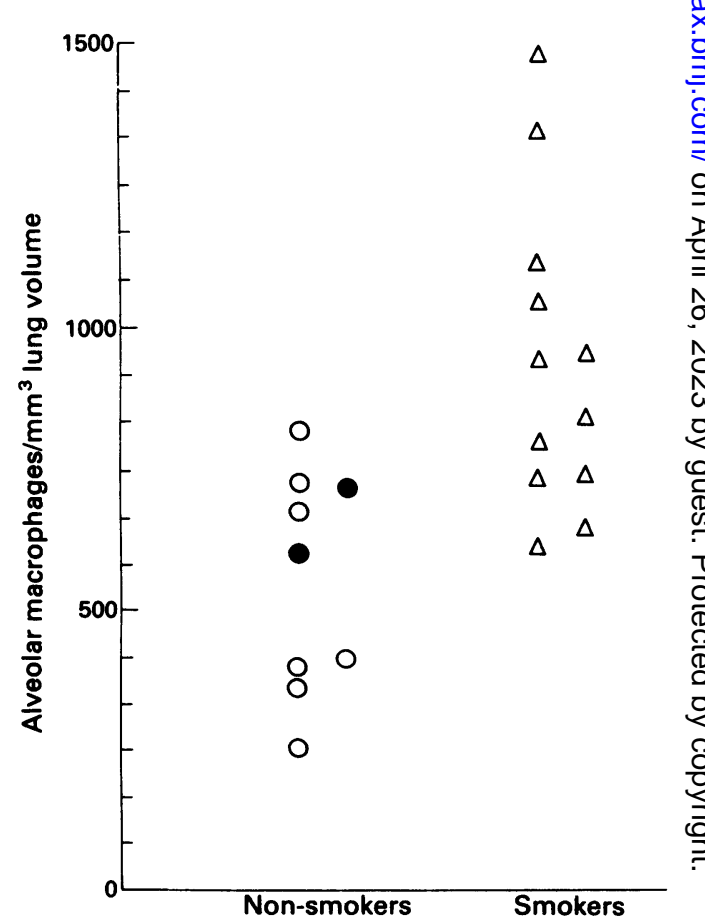

Figure 2 Alveolar macrophage number per $\mathrm{mm}^{3}$ lung volume in the two groups studied. The two ex-smokers are indicated by 0 . The smoking group shows a significant increase $(p<0.01)$ in alveolar macrophages per $\mathrm{mm}^{3}$ lung volume by comparison with the non-smokers. 


\section{Figure 3 Alveolar macrophage numbers per mm ${ }^{2}$ lung surface in the two groups studied. The two ex-smokers are indicated by 0 . The smoking group shows a significant increase $(p<0.001)$ in alveolar macrophages per $\mathrm{mm}^{2}$ lung surface by comparison with the non-smokers.}
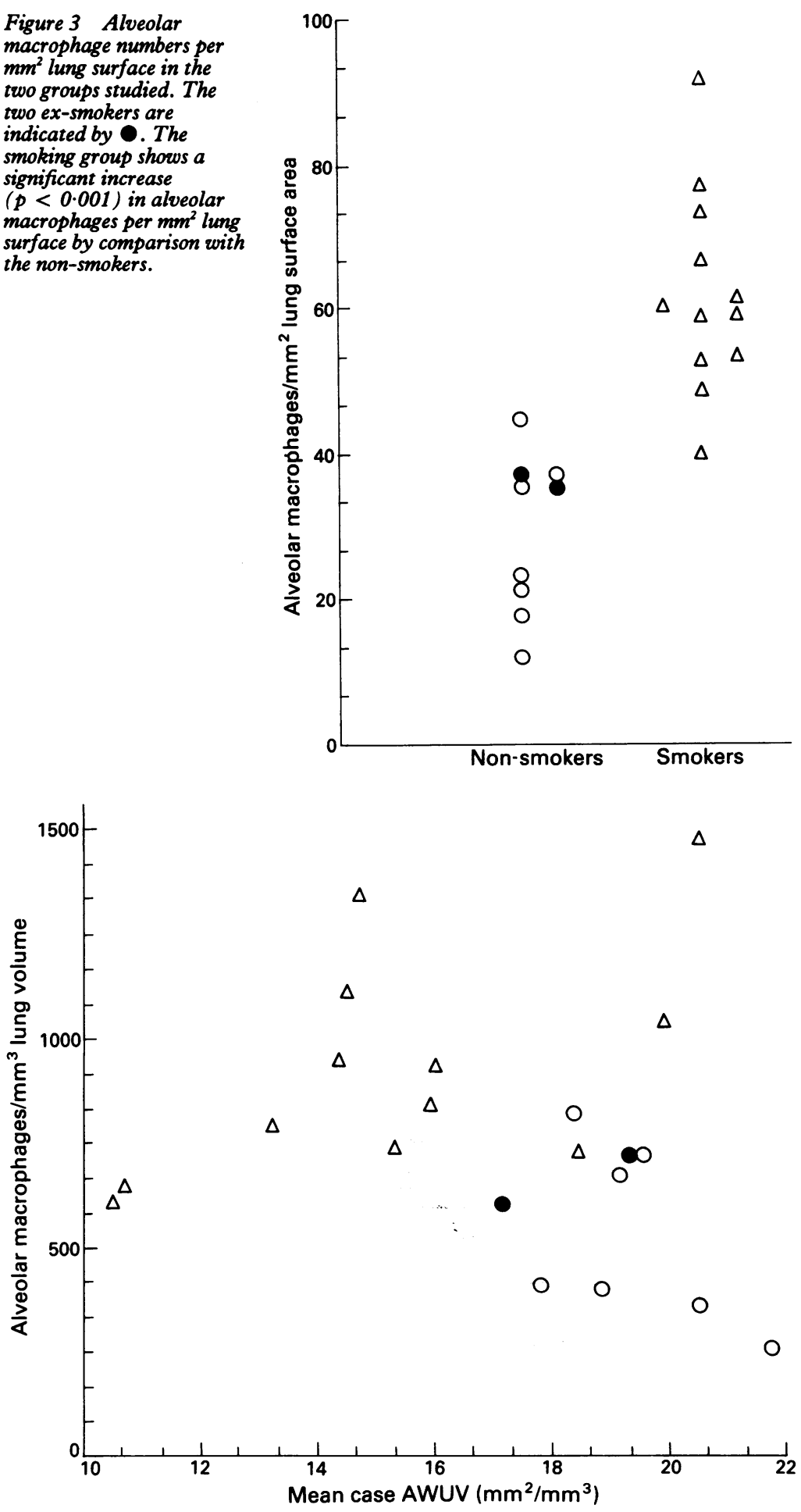

Figure 4 Alveolar macrophage number per $\mathrm{mm}^{3}$ lung volume in the cases studied as measured by airspace wall surface area per unit volume (AWUV). $\bigcirc$ Non-smoker; ex-smoker; $\triangle$ current smoker, indicating that a rise in alveolar macrophage number may occur in patients with no decrease in $A W U V$.

derived, on the assumption of a section thickness of $3 \mu \mathrm{m}$.

$$
\begin{aligned}
& \begin{array}{l}
\text { Alveolar } \\
\text { macrophages } \\
\text { per } \mathrm{mm}^{3}
\end{array} \\
& \text { mean macrophage score per } \mathrm{mm}^{2} \text { field } \times 1000 / 3 \\
& \text { mean macrophage diameter }
\end{aligned}
$$

This figure allows the extrapolation of the total number of alveolar macrophages in a lung volume, but as these cells relate in vivo to the lung surface and not the alveolar space the alveolar macrophage "density" or alveolar macrophages per $\mathrm{mm}^{2}$ lung surface was calculated:

\section{STATISTICS}

For all analyses of data a non-parametic MannWhitney test was used. Results are expressed as means and standard errors of the mean for all cases.

\section{Results}

MEASUREMENT OF LUNG STRUCTURE

Figure 1 illustrates the results of the measurements of lung structure in each of the 12 current smokers and nine non-smokers. The mean measured AWUV value $\left(\mathrm{mm}^{2} / \mathrm{mm}^{3}\right)$ was significantly lower $(p<0.05)$ for the current smokers $(15.35(0.91))$ than for the nonsmoking group (19.17 (0.46)).

\section{ALVEOLAR MACROPHAGE NUMBERS}

Figures 2 and 3 show the alveolar macrophage number per $\mathrm{mm}^{3}$ lung volume and per $\mathrm{mm}^{2}$ lung surface area. More alveolar macrophages were present in the smokers than in the nonsmokers when the numbers were expressed per $\mathrm{mm}^{3}$ lung volume (944.3 (77.8): $556(66 \cdot 2)$; $\mathrm{p}<0.01)$ or per $\mathrm{mm}^{2}$ lung surface $(62.0$ (3.8): 29.4 (3.7): $p<0.001$ ).

Figure 4 indicates how the number of alveolar macrophages varies with the measurement of lung structure. Smokers with a high AWUV are shown to have greater numbers of alveolar macrophages than nonsmokers with a similar AWUV.

\section{Discussion}

It is a matter of considerable surprise that no quantitative studies of the alveolar macrophage population in smokers and non-smokers have been carried out with tissue sections. Pathologists have long been aware that smokers have increased numbers of macrophages, often clumped together, adjacent to the respiratory bronchioles.

Previous studies of the alveolar macrophage population, using bronchoalveolar lavage, have been carried out in ignorance of the underlying lung architecture, which has not permitted quantification of the results. We have shown that with histological techniques numbers of alveolar macrophages can be related to meaningful measures of lung architecture.

Specimens clearly had to come from patients fit for thoracotomy, which introduced a selection criterion in that patients with evidence of appreciable respiratory failure were excluded. We deliberately included two ex-smokers (both had given up smoking more than six months previously) in the non-smoking group. Whereas structural damage, such as emphysema, that may result from smoking is not reversible when smoking ceases, possibly the alveolar macrophage population is more labile, though no data are available on this.

Our results support the view that there is a significant increase in the alveolar macrophage population in smokers, whether numbers are expressed per unit lung volume or per unit alveolar surface area. In vivo the alveolar macrophage must be related to the surface of the lung rather than its gas filled volume and it 
Alveolar macrophage numbers recovered in bronchoalveolar lavage fluid from smokers and nonsmokers in reported studies (calculated ratios indicating the relative increase in number of alveolar macrophages associated with smoking)

\begin{tabular}{lcll}
\hline & \multicolumn{3}{c}{ Macrophage yield from lavage fluid $\left(\times 10^{6}\right)$} \\
\cline { 2 - 4 } First author & Smokers & Non-smokers & Ratio \\
\hline Harris $^{6}$ & 46 & 10 & 4.5 \\
Warr $^{7}$ & 100 & 20 & 5.0 \\
Harris $^{8}$ & 125 & 27 & 4.6 \\
Rodrieguez $^{9}$ & 198 & 20 & 9.6 \\
Warr $^{10}$ & 67 & 14 & 4.8 \\
Low $^{11}$ & 72 & 11 & 6.7 \\
Demarest $^{12}$ & 121 & 15 & 8.2 \\
\hline
\end{tabular}

would therefore be more physiological to express the results in terms of surface area. Interestingly, our data show greater discrimination between the two groups when expressed in this way.

The smokers had an approximately twofold increase in alveolar macrophages in our study. This figure is considerably lower than the increases reported previously for cells obtained by bronchoalveolar lavage (table), where values ranging from around four to over nine have been reported. The reasons for the higher ratios in studies using bronchoalveolar lavage may relate to changes in lung architecture associated with smoking. We have observed, while performing lavages on surgical resection specimens, that the lavage fluid tends to be less confined to the area being lavaged and is more variably recovered in specimens with emphysema. Thus changes in lung architecture may result in smokers' macrophages being more easily "washed out." Another possible source of bias with bronchoalveolar lavage may relate to recovery of macrophages from the bronchial tree, which are excluded from histological sections and from selective "washout" from the proximal acinar unit.

Figure 4 shows the numbers of alveolar macrophages per unit lung volume in relation to the airspace wall surface area per unit volume. Smokers whose lung architecture, as assessed by AWUV, is similar to that seen in the non-smokers also have an increased number of alveolar macrophages. This suggests that the observed rise must be due to smoking and does not depend on any change in architecture.

We believe that if further data on the protective or pathogenetic roles of the alveolar macrophage are to be assessed meaningfully they must be quantitatively related to lung tissue. Expressing the number per unit lung volume or unit surface area provides a natural way of expressing the cell numbers in the context of variable abnormal lung architecture, something that previously has not been possible. This approach may be of value in the study of other inflammatory cells in the pulmonary parenchyma.

1 Brain JD. Lung macrophages: How many kinds are there? What do they do? [editorial]. Am Rev Respir Dis 1988; 137:507-9.

2 Reynold HY. Respiratory infections may reflect deficiencies in host defence mechanisms. Diseases Monthly 1985;31: $1-98$.

3 Sibille Y, Reynolds HY. Macrophages and polymorphonuclear neutrophils in lung defence and injury. State of the Art. Am Rev Respir Dis 1990;141:471-501.

4 Rennard SI, Hunninghake GW, Bitterman PB, Crystal RG Production of fibronectin by the human alveolar macrophage: mechanisms of the recruitment of fibroblasts to sites of tissue injury in interstitial lung disease. Proc Natl Acad Sci 1981;78:7147-51.

5 Gadeu JE, Pacht ER. The protease anti-protease balance within the human lung. Implications for the pathogenesis of emphysema. Lung 1990;168(suppl):552-64.

6 Harris JO, Swenson EH, Johnson JE. Human alveolar macrophages: comparison of phagocytic ability, glucose ultilisation and ultrastructure in smokers and nonsmokers. J Clin Invest 1973;49:2086-96.

7 Warr GA, Martin RR. Chemotactic responsiveness of human alveolar macrophages. Effects of cigarette smoking. Infect Immun 1974;9:769-71.

8 Harris JO, Olsen GN, Castle JR, Maloney AS. Comparison of proteolytic enzyme activity in pulmonary alveolar macrophages and blood leukocytes in smokers and nonsmokers. Am Rev Respir Dis 1975;111:579-86.

9 Rodrieguez RJ, White RR, Senior RM, Levine EA. Elastase release from human alveolar macrophages: comparison of smokers and non-smokers. Science 1977;198:313-4.

10 Warr GA, Martin RR, Sharp PM, Rossen RD. Normal human bronchial immunoglobulins and proteins. Effects of cigarette smoking. Am Rev Respir Dis 1977;116:25-30.

11 Low RB, Davis GS, Giancola MS. Biochemical analysis of bronchoalveolar lavage fluids of health human volunteer smokers and non-smokers. Am Rev Respir Dis 1978; 118:863-75.

12 Demarest GB, Hudson LD, Altman LC. Impaired alveolar macrophage chemotaxis in patients with acute smoke inhalation. Am Rev Respir Dis 1979;119:279-86.

13 Gould GA, MacNee W, McLean A, et al. CT measurement of lung density in life can quantitate distal airspace enlargement, an essential defining feature of human emphysema. Am Rev Respir Dis 1988;137:380-92.

14 Lamb D, Gillooly M, Farrow ASJ. Microscopic emphysem and its variation with age, smoking history and site within the lung. Am N Y Acad Sci 1991;624:339-41.

5 Gillooly M, Lamb D, Farrow ASJ. New automated technique for the assesment of emphysema on histological sections. J Clin Pathol (in press).

16 Aherne WA, Dunnill MS. Morphometry. London: Arnold, 1982:46-59.

7 McLean A, Lamb D. Morphometry of small airways in man J Pathol 1983;141:520.

18 Lindberg LG, Vorwerk P. Calculating volumes of transected bodies from two dimensional micrographs. Lab Invest 1970;23:315-8. 